\title{
The quantified older adult as design requirements for accessible wellbeing interventions
}

\author{
Andrew McNeill \\ PaCT Lab, Dept. of Psychology \\ Northumbria University, Newcastle \\ United Kingdom \\ andrew.mcneill@northumbria.ac.uk
}

\author{
Miglena Campbell \\ Dept. of Psychology \\ Teesside University \\ United Kingdom \\ Mia.Campbell@tees.ac.uk
}

\author{
Lynne Coventry \\ PaCT Lab, Dept. of Psychology \\ Northumbria University, Newcastle \\ United Kingdom \\ lynne.coventry@northumbria.ac.uk
}

\begin{abstract}
In this paper we describe how we engaged a group of healthy older adults in lifelogging and how we used their data to help design a healthy-aging intervention. 35 participants (mean age $=73.6$, age range 59-88, Men=15, Women $=20$ ) were tracked longitudinally for 18 months. Participants provided data at three time points. This involved the use of activity trackers, GPS trackers, and paper diaries. A number of well-established psychometric scales were also administered to gather standardised measures of health and wellbeing. While the data provides insight into the relationship between physical and social activity with regards to wellbeing, we aim to show that the nature of these relationships provide insight into how to design effective healthy-living interventions for older adults. By using the better predictors of wellbeing, we can target change in specific areas and assess change in those areas. While our sample is of relatively high-functioning older adults, we argue that understanding how they maintain wellbeing allows us to understand how to promote wellbeing amongst more inactive and frail older adults. Results showed the importance of specific types of social involvement such as meeting activity-group members and we propose that recommender systems should target these more important predictors of well-being.
\end{abstract}

\section{CCS CONCEPTS}

- Human-centered computing Empirical studies in ubiquitous and mobile computing • Social and professional topics $\sim$ Seniors

\section{KEYWORDS}

Older adults, life-logging, well-being.

\section{ACM Reference format:}

Andrew McNeill, Miglena Campbell and Lynne Coventry. 2019. The quantified older adult as design requirements for accessible wellbeing

Permission to make digital or hard copies of all or part of this work for personal or classroom use is granted without fee provided that copies are not made or distributed for profit or commercial advantage and that copies bear this notice and the full citation on the first page. Copyrights for components of this work owned by others than the author(s) must be honored. Abstracting with credit is permitted. To copy otherwise, or republish, to post on servers or to redistribute to lists, requires prior specific permission and/or a fee. Request permissions from Permissions@acm.org. PETRA '19, June 5-7, 2019, Rhodes, Greece

(C) 2019 Copyright is held by the owner/author(s). Publication rights licensed to ACM.

ACM ISBN 978-1-4503-6232-0/19/06...\$15.00

https://doi.org/10.1145/3316782.3321533 interventions. In Proceedings of PETRA (PETRA'19). ACM, New York, NY, USA, 10 pages. https://doi.org/10.1145/3316782.3321533

\section{Introduction}

In the past 40 years biopsychosocial models of health have begun to paint a vastly more complex picture of health as the outcome of the dynamic interactions between body, mind, and environment rather than merely the absence of disease. When it comes to older adults' health, behavioural efforts are focused around remaining physically, cognitively and socially active past retirement age. As part of the ACANTO project we wanted to build a recommender system to encourage such activity and to promote well-being among older adults. Our basic approach was to begin by understanding what activities are most beneficial to older-adult well-being so that we had a clear idea of how to design a recommender system to promote well-being. Firstly, to show the importance of designing for wellbeing, we survey some of the literature that shows the benefits of physical and social activity. We then move on to consider how older quantification of older adults could be used to design a recommender system for well-being.

\subsection{Benefits of physical activity}

Extensive research shows the benefits of physical activity for older adults. Higher physical activity predicts reduced depression [51], reduced cognitive impairment [4], and reduced fear of falling [47]. It also potentially results in increased quality of life scores [35]. A review paper looking at a variety of studies found that increased physical activity leads to improved life satisfaction and reduced health problems [48]. This review also found that the effects of physical activity were mediated by the enjoyment of such activities, increases in self-efficacy, and increases in self-satisfaction. Effects were moderated by sudden decreases in physical activity, their perception of the value of physical activity, and the influence of important others [48].

\subsection{Benefits of social activity}

Similarly, extensive research shows the benefits of social involvement for older adults and the damaging effects of loneliness [30]. While physical activity is beneficial, the integration of social aspects into such activities is important for optimising beneficial outcomes [40]. Even apart from physical activities, social activities by themselves are very important. By fulfilling fundamental needs for belonging [5], older adults find psychological satisfaction in 
joining social groups (e.g. interest-groups). These groups then provide many benefits in terms of psychological well-being for older adults [28, 33]. Recent developments in technology mean it is now possible to remain connected with an online social network even if one is physically unable to participate socially.

\subsection{Life-logging for wellbeing}

According to the Biopsychosocial model of health [21, 38] both biological dynamics (ones that capture the physical elements of the body) and interpersonal dynamics (ones that refer to actual or perceived social contacts) contribute to wellbeing interactively. Indeed, empirical evidence suggests social networks and role-related conflicting demands, may be important drivers of physical activity [7] and that even having someone in close proximity is a predictor of higher physical activity [42]. Given the importance of physical and social activity both separately and together for well-being, it is helpful to look for sources of such data in the lives of older-adults. With the proliferation of sensors that enable individuals to track various aspects of their lives, scholars have begun to talk about the quantified self - a picture of the self, built through longitudinally collected activity data. The notion of the quantified self involves building and understanding of one's own activity patterns and baseline measures [53]. Such data can be collected via self-report measures [6] but is more often collected through the use of digital devices, e.g. fitness trackers, GPS monitors, cameras and heart-rate monitors (to give only a few examples). A great number of healthrelated parameters discussed above could be potentially tracked, these can be classified into four broad domains [49]: physical states (e.g., steps), psychological states (e.g., mood), social interactions (e.g., Facebook "likes") and the environmental context they are in (e.g., pollution, pollen count). Together these domains comprise the source material for life-logging.

A body of literature suggests that older adults see value in keeping track and have been involved in pen-and-paper life-logging even before the arrival of digital technology [24]. Many older adults see great benefits in logging at least some aspects of their lives that they are interested in [11]. Wearable activity trackers are also gaining popularity, even though it is not clear if their default activity goals are suitable for older adults in terms of intensity [54]. Wearable trackers are a feasible consumer option for older adults as they are easy to use and affordably priced [3]. Wearables have also been shown to have high criterion validity in measuring walking in older adults [45]. Given the widespread use of activity trackers and their usefulness in assessing physical activity, we wanted to see if such data could give insight into how to design for well-being.

\subsection{The ACANTO recommender system and social network}

We began work on a digital ageing-well intervention as part of a larger project for assisted living. The ACANTO social network is designed primarily with older adults in mind. The system will operate around understanding users' interests and hobbies and its main function will be to recommend events and activities based on those interests/hobbies. The system will also connect older adults in groups or social circles with common interests. Recommendations will provide comprehensive information on events including logistical aspects, information about other attendees as well as means for planning, goal setting and tracking of social, cognitive and physical involvement. For example, a recommended event could be, "There is a meeting of the local history group in town, tomorrow at 2pm" or "There is a demonstration of gardening skills at the local allotments, tomorrow at 3pm.” Ultimately, the purpose of the social network is to provide daily opportunities for keeping active in old age and thus, delaying the onset of geriatric frailty. While there are other components of the project (such as an assisted walker), we focus here on the recommender-system and social network aspects of the project. To design these components effectively, we needed to be able to identify the best activities for wellbeing in order to focus on them in the recommender system and social network.

\subsection{Previous work on activity recommendation systems}

While there are many different types of recommender systems (RS), we are interested in recommender systems for events specifically. With the advent of Event-Based Social Networks [39] research has increasingly focused on how to recommend events to users that they will enjoy. One of the unique challenges of eventbased RS is that events occur only once and it is not often possible to recommend them again if they were previously enjoyed (“content-based RS" [9]). Additionally, given the variables involved in distinguishing different events that have some similarities (e.g. watching football and playing football are both "football" events but very different in nature), it can be very difficult to recommend events to users. However, the research shows that when algorithms are compared a combination of content-based recommendations and collaborative filtering is more effective than other approaches [15, 20, 37]. Such an approach recommends events to users if they are similar to past events that similar users enjoyed.

From our perspective, these approaches are valuable in that they seek to recommend events to users that they enjoy by using data about their preferences and those of similar others. However, while that is part of our goal (facilitating enjoyment), we also want to recommend events to users that will be good for their well-being. This requires not only a recommender system that generates likeable recommendations, but one that generates recommendations based on prior knowledge about what aids wellbeing.

\subsection{Behaviour change and wellbeing interventions}

Our aims for the project are drawn from research into behaviour change that suggests interventions be (1) easy for users to perform, (2) attractive to users, (3) social and (4) timely [27]. When designing an intervention to improve the well-being of older adults by recommending social and physical activities we therefore need to ensure that the activities recommended are: (1) easy and reflect the kinds of activities older adults might normally perform. This requires gathering data about the kinds of activities healthy older 
adults regularly perform. (2) Attractive to users in that they appeal to users' personal interests. (3) Social in that they are situated within the user's network of friends and involve that network in the activity recommendations. (4) Timely in that the recommendations occur when the users are likely to take part in the activities and recognise the temporally situated nature of activity-performance. These behaviour-change requirements form the basis for our objects of interest in this study: we look at the kinds of activities of regular activities older adults perform that are good for well-being (easy and attractive), we look at the kinds of social contact that are good for well-being (social), and we look at the times at which older adults perform regular activities (timely).

\subsection{Aims and Objectives}

The present study aimed to use life-logging data from a cohort of older adults to understand the sources of wellbeing in older adults to design an effective recommender system for social and physical activities. Based on the literature we reviewed we made the following predictions:

- $\quad$ There will be a positive relationship between physical activity and well-being

- $\quad$ There will be a positive relationship between social activity and well-being

- $\quad$ Different types of physical (e.g. walking or exercise) and social (e.g. religious or interest-group) activity will have different effects on well-being

- $\quad$ Over time there will be changes in well-being as a result of changed social or physical activity

In terms of design, our goal was to see if tracking data from older adults would provide data that let us predict the sources and patterns of wellbeing. Then, using the data about what is most beneficial for well-being, we seek to develop a system that can support that wellbeing. In an integrated system, the continuous input of lifelogging data would monitor the wellbeing of the users and this could then be used as the basis for recommending activities for the user that are of demonstrable benefit. While we draw on aggregated data in this study in order to make statistical inferences, individual data collected over a longer period of time would provide the basis for a personalised recommendation system that would consider the accessibility of an individual user.

\section{METHOD}

\subsection{Design}

The study used a longitudinal design across an 18 month period. The life-logging data comprised of both data collected via sensors (GPS and activity trackers) and via self-report (diaries and questionnaires).

\subsubsection{Participants}

35 participants (mean age $=73.6$, age range 59-88, Males=15, Females= 20) were recruited in England from the North East Age Database held at [NAME] University. While the sample size was

\footnotetext{
${ }^{1}$ https://misfit.com/go/flash

${ }^{2}$ https://misfit.com/go/shine
}

relatively small, gaining large samples for longitudinal studies is a challenge. Participants all lived independently in the community and were mostly retired or semi-retired. They were almost entirely White British and possessed a variety of qualifications that indicated a generally high level of education. Most participants were in relatively good health although one participant was blind and one had chronic fatigue syndrome.

While a less healthy sample with lower well-being would have been helpful in this study to see if well-being scores could be improved for them, the realities of gaining participants in this demographic meant that it is difficult to get access to those participants for a longitudinal study.

\subsubsection{Devices}

The Qstarz BT-Q1000XT GPS tracker was used to track the participant's location. From this was derived speed of walking and distance travelled between points. The device was generally reliable, and participants had to charge it every day or two. Data was transferred directly via a USB cable for analysis. 11 GPS trackers were used in the study meaning that not every participant used a GPS tracker at each time point.

Misfit activity trackers (Flash ${ }^{1}$, Shine ${ }^{2}$ and Shine $2^{3}$ ) were worn to measure daily steps. The Misfit trackers were chosen because of the long battery life (several months) and while the Flash was used initially, problems with water-resistance led to the use of the Shine (which had problems with falling out of the watch strap) and the Shine 2. The Misfit API allowed data to be accessed for analysis.

\subsubsection{Psychometric instruments}

While life-logging data showed at a granular level the activities of participants and changes taking place, we also used standardised psychological measures in order to understand the wellbeing of participants. These were measured via an annual questionnaire. Key measures we used were:

- Falls Efficacy Scale International Short form [34] - to assess fear of falling

- $\quad$ Geriatric Depression Scale $[1,56]$

- The Brief Resilience Scale [50] - to assess psychological resilience

- $\quad$ Personal wellbeing index [32]

- de Jong [26] - a measure of loneliness

- $\quad$ EuroQL 5D 5L (Quality of Life) [31] - a health-related quality of life measure

- $\quad$ UAB life-space assessment [46] - an index of the general radius in which a participant lives their life

Daily diaries completed by participants at set intervals across the 18 months. These diaries contained the following measures:

- $\quad$ Mood (PANAS scale) [55]

- Social or physically active activities (qualitative response)

- $\quad$ People you met with today outside of people in your home (qualitative response)

- $\quad$ Perception of physical activity (Likert 5-point scale)

- $\quad$ Perception of social involvement (Likert 5-point scale)

${ }^{3}$ https://misfit.com/go/shine2 
- Perception of cultural involvement (Likert 5-point scale)

- Perception of creative-activity involvement (Likert 5point scale)

- Life-space zones (5 questions derived from UAB lifespace scale [46])

- $\quad$ Other reflections on the day (qualitative response)

\subsubsection{Procedure}

Participants were asked to commit to participation for the duration of the project (with the right to withdraw for any reason). Baseline measurements and demographic data were collected in the beginning of testing via the questionnaire. Follow-up measurements were collected at two further time points. Each questionnaire took around 30 minutes to complete.

At each visit, participants were provided with a paper diary and asked to record their social and physical activities for a two-week period. One participant provided answers to the diary questions via a daily telephone call. Participants then returned to the University after two weeks to return the diary. Participants were also provided with a GPS tracker and an activity tracker. Written instructions on how to use the devices were made available to participants. These devices were carried/worn by participants for a two-week period (to coincide with the diary task). This meant that a wealth of lifelogging data was provided by participants during each two-week period. The two-week period did not necessarily overlap with that of other participants meaning that GPS trackers could be returned and provided to other participants. Details of the dates of data collection can be found in Table 1 .

Table 1: Timeframe of data collection

\begin{tabular}{c|cc|c} 
Objects & Time 1 & Time 2 & Time3 \\
\hline $\begin{array}{c}\text { Questionnaire } \\
\text { (annual) }\end{array}$ & Jan-Jul & - & Mar-Jun Year 2 \\
$\begin{array}{c}\text { Yiary, GPS and } \\
\text { activity tracker (2- } \\
\text { week period) }\end{array}$ & Jan-Jul & $\begin{array}{c}\text { Aug- } \\
\text { Year 1 }\end{array}$ & Mar-Jun Year 2 \\
Year 1 &
\end{tabular}

\section{RESULTS}

In these results, we document how quantified-self data can provide insight into wellbeing in the older adult participants. The two main dependent measures of wellbeing were the EQ-5D-5L scale (QoL; measuring health-related quality of life; [31]) and the Personal Wellbeing Index (PWI; measuring overall wellbeing; [32]). Both measure slightly different aspects of wellbeing. For the analysis we tested for relationships between the quantified measures of physical and social activity and the two wellbeing measures.

\subsection{Descriptive statistics}

The participants in the study showed a relatively high level of physical activity as indicated by daily steps (M (mean) $=6829$ steps; SD (standard deviation $)=3499$ ) and a relatively high level of self-assessed social involvement on a daily basis $(\mathrm{M}=3.68$; $\mathrm{SD}=.68$ - where 3 represented "neither involved nor uninvolved" and 4 represented "somewhat involved"). Personal wellbeing was also relatively high $(\mathrm{M}=7.54, \mathrm{SD}=1.41$ - assessed on a scale of 0 10 where 10 was equal to completely satisfied with life). Health- related quality of life (QoL) was also relatively high $(\mathrm{M}=4.36$, $\mathrm{SD}=.54$ - measured on a 5-point scale). Given the relative lack of diversity in these scores (apart from steps), we did not consider it warranted to split participants into groups based on scores of social or physical activity involvement to compare differences between high and low scoring participants. We did consider splitting participants based on step counts, but the consequently small sample size for each group did not produce significantly different outcome scores.

Despite the use of a small sample size in the analysis, the independent variables used in analysis (steps, distance travelled, social involvement) represent averages of data gathered across the different time-points and across multiple days. This means that random error in the IV measurements is reduced.

\subsection{Steps indicate wellbeing}

The data showed a significant, moderate, and positive correlation between steps and Quality of Life (EQ-5D-5L) where r (Pearson's correlation coefficient $)=.398, \mathrm{p}$ (significance level $)=.027$. In a regression model, we also controlled for the effect of social involvement (self-reported). The model was significant $F(2,30)$ $=3.842, \mathrm{p}=.034)$ and $\mathrm{R}^{2}$ (variance explained) $=.215$. Mean daily steps were the only significant predictor in the model ( $\beta$ (strength of predictor) $=.368, \mathrm{p}=.038$ ). This shows the usefulness of step count data in predicting health related quality of life.

\subsection{Distance travelled indicates wellbeing}

Using GPS data, we were able to track the location of older adults when they left their homes. By calculating the distance between two consecutive GPS points, we were able to calculate the speed of travel by dividing the distance by the time-difference between the two points. The total distance travelled, average speed travelled (including speed in vehicles), and average walking/running speed travelled ( $<7 \mathrm{~km} / \mathrm{h}$; based on findings in [52]) were calculated for each day and matched with corresponding entries in the participant diaries.

Positive mood was related to all three of the variables: distance $(\mathrm{r}=.107, \mathrm{p}=.012)$, average speed $(\mathrm{r}=.096, \mathrm{p}=.024)$, and average walking speed $(\mathrm{r}=.179, \mathrm{p}<.001)$. We also looked at the relationship of this GPS data (averaged over the course of the project) with selfreported measures in the questionnaires (averaged over the course of the project). Distance travelled is related to improved QoL scores ( $\mathrm{r}=.446, \mathrm{p}=.015, \mathrm{n}=29$ ), increased wellbeing (PWI; $\mathrm{r}=.452, \mathrm{p}=.014$, $n=29)$, and reduced fear of falling $(r=-.463, p=.011, n=29)$. Interestingly, distance travelled is a stronger correlate of quality of life than steps, indicating the value of being able to use different predictors of wellbeing, especially if steps is not an appropriate measure for some users given specific disabilities.

\subsection{Social involvement indicates wellbeing}

Self-reported social involvement (via diaries) was not significantly correlated with either personal wellbeing (PWI) or quality of life. However, the latter was almost a significant correlation $(r=.339$, $\mathrm{p}=.05)$. Certainly, other aspects of wellbeing such as depression $(\mathrm{r}=-.413, \mathrm{p}=.015)$ and loneliness $(\mathrm{r}=-.626, \mathrm{p}<.001)$ were negatively 
correlated with social involvement indicating the positive effects of social involvement in reducing these specific issues.

Given these associations between physical and social activity and the impact on wellbeing (and/or aspects thereof), we proceeded to investigate what types of physical and social activity were of most benefit to older adults. At this point, the life-logging data became very useful. Participants recorded what types of activities they were involved in, and what types of people they met with in the diaries.

\subsection{Different types of activity provide different benefits}

In the diaries, participants rated the extent to which they were involved in physical, social, cultural and creative activities. This provided a broad-brush approach to understanding the benefits of different types of activities. We found that cultural ( $r=.342, \mathrm{p}=.047$, $\mathrm{n}=34)$ and creative $(\mathrm{r}=.372, \mathrm{p}=.031, \mathrm{n}=34)$ activities have significant positive relationships to quality of life. Effects of social involvement are more prominent for other variables: psychological resilience (being able to cope with adversity) is stronger with increased social activity $(r=.46, p=.006, n=34)$ and depression is reduced $(\mathrm{r}=-.413, \mathrm{p}=.015, \mathrm{n}=34)$. Perceived physical activity is also linked to reduced depression $(r=-.445, p=.008, n=34)$. All the activities were linked to reduced loneliness but the relationship with social involvement, unsurprisingly, was strongest $(r=-.626$, $\mathrm{p}<.001, \mathrm{n}=34$ ).

While this data provides a broad overview of benefits, we were interested in the effects of specific types of activities. Word frequency queries of this data enabled us to quantify references to different types of activities and social contact and calculate the psychological effects of such activities/contacts. The different types of activities identified in the diaries are listed in Table 2. Only activity-related nouns were identified, and stemmed words were not counted separately (e.g. walk and walking). Conceptually linked words were also collapsed (e.g. “coffee” and "lunch”). Frequencies less than 40 were not counted due to the lack of statistical power in smaller numbers. Using these different categories of activity, we were able to look at whether taking part in such activities more frequently over the course of the study was related to specific benefits overall. Participants who took part in an activity on a given day were scored 1 for that variable, while those who did not take part received 0 . The average of participation across all the days was then taken and used as an index of how often the participant took part in a given activity. Relationships between each activity and various outcomes were then tested. The findings are presented in Table 2.

Table 2: Activities and benefits

\begin{tabular}{c|c} 
Activity & Benefits/Effects \\
\hline Walking (464) & $\begin{array}{c}\text { Participants feel more active }(\mathrm{r}=.216, \\
\mathrm{p}<.001)\end{array}$ \\
Shopping (200) & $\begin{array}{c}\text { Associated with higher life-space } \\
(\mathrm{r}=.107, \mathrm{p}<0.005)\end{array}$
\end{tabular}

\begin{tabular}{|c|c|}
\hline Activity & Benefits/Effects \\
\hline $\begin{array}{l}\text { Having coffee (131) } \\
\text { or lunch (85) }\end{array}$ & $\begin{array}{l}\text { Associated with meeting friends } \\
(\mathrm{r}=.253, \mathrm{p}<.001) \text {, higher perceived } \\
\text { social involvement }(\mathrm{r}=.201, \mathrm{p}<.001) \\
\text { and higher life-space }(\mathrm{r}=.154, \mathrm{p}<.001)\end{array}$ \\
\hline Dog walking (123) & $\begin{array}{l}\text { Higher feelings of being active } \\
(\mathrm{r}=.117, \mathrm{p}<.001) \text {, higher life-space } \\
(\mathrm{r}=.162, \mathrm{p}<.001) \text {, feeling socially } \\
\text { involved } \mathrm{r}=.068, \mathrm{p}=.026) \text {. Associated } \\
\text { with meeting friends ( } \mathrm{r}=.230 \text {, } \\
\mathrm{p}<0.005) \text { and neighbours ( } \mathrm{r}=.06 \text {, } \\
\mathrm{p}=.046) \text {. Higher average walking } \\
\text { speeds ( } \mathrm{r}=.116, \mathrm{p}=.006) \text { - via GPS } \\
\text { tracker. }\end{array}$ \\
\hline Gardening (94) & $\begin{array}{l}\text { Slight increase in likelihood of } \\
\text { meeting neighbours }(r=.081, p=.007)\end{array}$ \\
\hline $\begin{array}{l}\text { Exercise (e.g. } \\
\text { “gym”, 43) }\end{array}$ & $\begin{array}{l}\text { Higher perceptions of being active } \\
\qquad(\mathrm{r}=.109, \mathrm{p}<.001)\end{array}$ \\
\hline $\begin{array}{l}\text { Religious activity } \\
\text { (e.g. “church”, 45) }\end{array}$ & $\begin{array}{l}\text { Higher likelihood of meeting friends } \\
\text { (r=.114, } \mathrm{p}<.001) \text { and feeling socially } \\
\text { involved ( } \mathrm{r}=.092, \mathrm{p}=.003) \text {. Lower } \\
\text { average walking speed ( } \mathrm{r}=-.113 \text {, } \\
\mathrm{p}=.008) \text { - via GPS tracker. }\end{array}$ \\
\hline
\end{tabular}

The data shows the diverse benefits of different types of activities. It also highlights activities that are particularly beneficial such as dog walking and meeting a friend for coffee or lunch. From a design perspective, knowing the benefits of such activities helps to guide the design of systems to recommend activities to improve wellbeing.

\subsection{Different types of social involvement have different benefits}

Similar to the process for identifying different types of activities, we also identified different types of social contact. In the diary we asked participants to identify who they met with during the day. We then observed the frequencies of words used to identify people they met to enable coding for the following types of people: friends $(n=374), \quad$ staff/assistants $\quad(n=257)$, neighbours $\quad(n=122)$, members/group ( $\mathrm{n}=141)$, family $(\mathrm{n}=186)$.

We found that meeting a friend was associated with higher perceptions of feeling active $(\mathrm{r}=.169, \mathrm{p}<.001)$, being socially involved $(r=.322, \mathrm{p}<.001)$ and higher life space $(\mathrm{r}=.183, \mathrm{p}<0.005)$. By contrast, meeting a neighbour had no significant correlations with any of these.

Meeting family members was associated with significantly higher moods ( $\mathrm{r}=.114, \mathrm{p}<.001$ for overall PANAS scores: Positive minus negative scores) and led to higher feelings of social involvement $(\mathrm{r}=.126, \mathrm{p}<.001)$. It is important to remember the benefits of family connectedness for older adults, and while this is not available equally to all older adults, cannot be forgotten about as an important source of well-being. 
While the benefits of family connectedness via social contact are important, perhaps equally or even more important is connectedness with interest groups of various kinds. These kinds of groups include writing groups, pantomime groups, litter-picking groups, badminton groups, reading group and many more (all identified in the diaries). These have benefits for daily mood $(\mathrm{r}=.177, \mathrm{p}<.001$ for overall PANAS), perceptions of social involvement $(\mathrm{r}=.223, \mathrm{p}<.001)$, cultural involvement $(\mathrm{r}=.214$, $\mathrm{p}<.001)$, and creative activity $(\mathrm{r}=.14, \mathrm{p}<.001)$. This involvement also has a small correlation with increased life-space $(\mathrm{r}=.118$, $\mathrm{p}<.001$ ). Much literature has pointed out the benefits of belonging to such social groups, and the evidence from this study shows the variety of benefits and that such effects are stronger than even family connectedness.

Perhaps surprisingly, the benefits of social contact are not simply for common-bond or common-identity groups like families or clubs respectively, but even relatively minor social contact with shop assistants and other members of staff (at restaurants, banks, cinemas, etc.) have benefits for older adults. Meeting staff members was associated with small but significant benefits on positive mood $(\mathrm{r}=.102, \mathrm{p}=.001)$ and while it did not lead to feelings of more social involvement, led to increased life-space scores ( $\mathrm{r}=.137, \mathrm{p}<.001)$.

We also investigated the relationship between meeting different types of people and scores across a range of measures in the questionnaires (averaged across the course of the project). Who a person reported meeting up with in their diaries was related to their well-being. People who more frequently reported meeting staff or family did not report higher well-being scores, but those who reported meeting group members more often also reported higher levels of well-being in the questionnaires $(r=.391, \mathrm{p}=.022, \mathrm{n}=34)$. Also, intriguing was the finding that only meeting with group members seemed to act as a buffer against depression ( $\mathrm{r}=-.405$, $\mathrm{p}=.017, \mathrm{n}=34$ ). Likewise, loneliness was also reduced among those who more frequently met with group members $(\mathrm{r}=-.420, \mathrm{p}=.013$, $\mathrm{n}=34$ ) and this relationship was also seen for those who met with friends ( $r=-.465, p=.006, n=34$ ). These findings speak to the value of social meetings for older adults, but particularly to the importance of meeting with members of interest groups. Perhaps the combination of having a shared sense of purpose and social support provides this importance source of well-being.

\subsection{Quantified activity data show temporal patterns}

Understanding the activity patterns of older adults is not simply about understanding what activities and meetings are most beneficial, but it helps to understand the temporal pattern of activities. Doing word frequency queries on the diaries allowed us to see patterns across the seasons. We then plotted these different activities on a line graph as illustrated in Figure 1. While we need to be cautious about generalising too much from this data, the graph shows that seasonal variation does affect activity choices. Some activities tend to dip (a U-shape) during the spring-summer months where there is better weather: shopping, exercise and going to religious buildings. Other activities show an increase during the spring-summer months (an inverted U-shape): walking, going for coffee/lunch and gardening. Understanding such variations is important for designing a recommender system that can offer appropriate activities for different times of the year.

The data also shows evidence of weekly patterns. Categorising activities by day of the week when they are undertaken shows these patterns. Some activities occur most frequently at the weekend: gardening, religious service attendance, and shopping. Others are

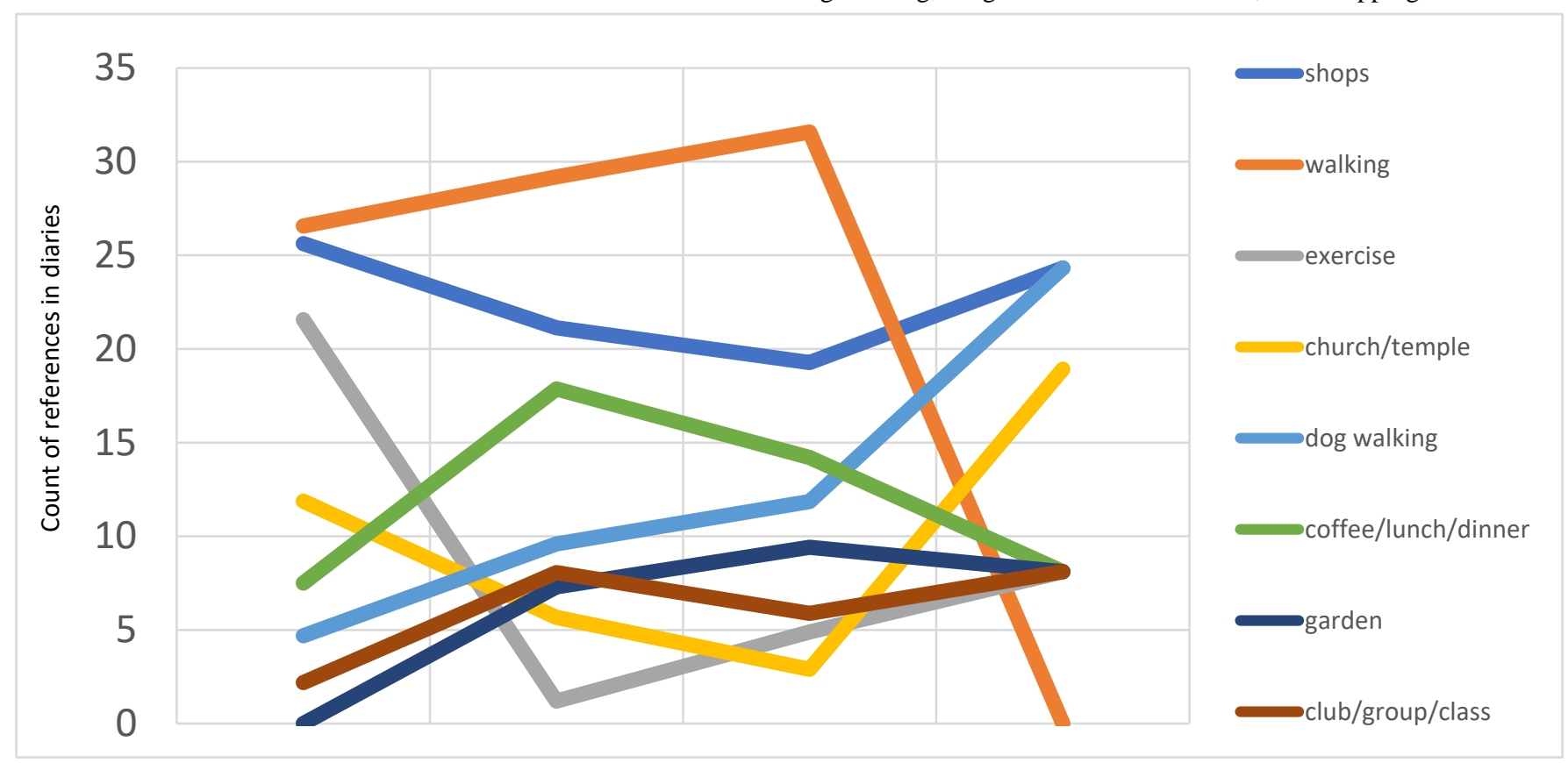

Figure 1: Line graph showing seasonal variation in activities 
more likely to be undertaken Monday-Friday: Exercise and dog walking (including Sundays). Looking at GPS distance patterns across the week shows that distance peaks on Wednesdays (midweek) and Saturdays. The fact that some activities are more frequent at the weekend and that distance travelled peaks on a Saturday suggests that while older adults might be retired, they do not necessarily spread their activities across the week. Indeed, the presence of things like markets and other events at weekends probably incentivises as much activity at the weekend as it does for non-retired people. Translating this to design requirements suggests that when encouraging activity in older adults, weekly patterns need to be considered.

\section{DISCUSSION}

The present study aimed to track a cohort of 35 older adults for 18 months to quantify their trajectory in terms of changes in activity levels and in a number of psychosocial parameters. We did this to generate the foundations for designing a system that recommends wellbeing enhancing activities for older adults. We anticipated that wellbeing and quality of life would be positively impacted by regular participation in physical, social and cultural activities. This was indeed true for our sample, as distance travelled, daily steps and social involvement were all indicators of wellbeing. This was also consistent with previous research, which ties participation in social and physical activities to health benefits [28, 33, 51]. Social and physical activities are in daily life more often than not intertwined and it is possible that their effects on wellbeing are synergistically enhanced. While it is clear that some benefits may come from the physical act of walking (as indicated by the positive correlations of steps and distance travelled with wellbeing) others come from the likelihood of meeting friends and relatives when travelling, rather than from the physical distance from one's home. We also found that mood was positively associated with walking speed. This is consistent with a whole body of literature, which links these two variables [47, 48, 51]. However, the relationship between them is far from clear and establishing causation is difficult. In our case, it is hard to show whether walking speed had caused increases in positive mood, or whether participants found themselves walking in a more upbeat manner because they were in a good mood to begin with. It is important not to draw incomplete inferences or make premature suggestions on this issue, as high walking speed is associated not only with benefits for older adults, but also with costs, such as risk of falls and injuries [44].

Once again, it is important to exercise caution when talking about wellbeing in the present context. Individuals who volunteered for our study were often relatively high functioning, mostly affluent and very active in social, cognitive and physical terms. A large proportion of our cohort had multiple hobbies (e.g. reading, gardening, geology, flying aircrafts, politics, art, art history, volunteering, and lifelong learning) and were involved in at least one physical activity or sport (e.g. golf, badminton, walking football, swimming, dancing, going to the gym, yoga etc.). Additional analyses suggested that the distribution of comorbidities of this group was skewed towards fewer age-related disabilities and health problems. As such, it is not too surprising that in this self- selected sample age-associated decline of activities, capacities and psychological parameters was not observed within the 18 months of tracking we conducted. Literature on ageing well suggests that wellbeing remains preserved or increases with age [12-14, 36]. Our findings are consistent with this notion, however it is important to keep in mind that in comparison to the general population, our cohort's scores were once again skewed towards higher than average wellbeing. The current study does not bring any additional insights on why wellbeing in this sample was higher than average to begin with and improved in time, however one explanation suggests that older adults may consciously and deliberately make a decision to be happy, that is they employ cognitive strategies to maximise their happiness [8].

The present study also demonstrated that different types of activity provide different benefits for participants. For instance, feeling socially involved was positively associated with feeling resilient and negatively associated with feeling depressed. Involvement in cultural and creative activities was linked to increased quality of life, and perceived physical activity was linked to reduced depression. Walking and going to the gym was associated with participants feeling more physically active, going shopping was associated with a larger life space and religious activities was associated with feeling more socially involved. These finding informed the design of the system in a way which will ensure that it recommends to users building a rich tapestry of diverse activities to obtain a full spectrum of health benefits.

One activity that produced positive changes on multiple parameters was dog walking. It was positively associated with expanding participants' life space and was associated with them feeling more physically and socially active. Consistent with previous literature [17] dog walking indeed facilitates increases in physical activity. However, we also found that dog walking was positively associated with meeting more people, i.e. more friends and neighbors were encountered on days when participants took their dog out for a walk. It is not quite clear whether dog walking was the cause of meeting more people, however in terms of intervention development it is evident that by targeting the physical domain, one may inadvertently affect the social and vice versa. It is important to note that when it comes to dog walking other variables may also be at play. For instance, cognitive and emotional factors may also be implicated, as it has been established that dog ownership can carry unique cognitive and emotional benefits to older adults [41]. Recommendations regarding becoming involved in owning a dog should be made with caution, as this is also associated with certain costs [43].

Our results also show that different types of social interaction had different effects on wellbeing. While meeting family members was positively associated with good mood and facilitated feelings of social involvement, participants also reported a great deal of positive impact from socialising in groups based on interests. Complementing some research [16], which suggests that expanding the number of confidants in older adults' social circle is related to psychological benefits, we found that even very low quality social interactions (e.g. with retail staff) contributed to mood improvements. This has an important implication for those 
older adults, for whom family connectedness or participation in interest groups are not available. It means that seeking any form of social involvement, even if it is relatively transient or fleeting, is worthwhile and may prevent broad-based morbidity and mortality associated perceived social isolation [10, 29]. However, given the distinct benefits of engaging with interest groups, we have designed our system to facilitate the engagement of interest groups. By involving the leaders of interest groups in disseminating events via the system, we aim to focus our attention on activities that promote most wellbeing for users.

Our results also indicated a temporal pattern to participants' behaviours, e.g. they were most likely to do gardening and shopping on weekends and exercising was a weekday activity, which peaked on Mondays and reached its lowest points at weekends. This indicates that such behaviours may largely be habit-driven and stem from automaticity formed most likely prior to participants' retirement. Designing for such temporal variation means that the system will need to adaptively recommend different activities for different days and seasons. Understanding, for example, that gardening is more common at weekends means that the system can encourage such activities at or for such relevant times. With regards to exercise patterns, Psychological Reversal theory [2] suggests that behaviours such as exercising are more congruent with goal-oriented ("telic") states of mind. Leisurely activities are conversely more easily embedded in playful and impulsive 'ludic' mindsets. This theoretical explanation may suggest that older adults see exercising as a conscious and conscientious effort towards health maintenance, rather than primarily a pleasure pursuit. Previous research suggests that older adults report various health benefits from exercising [23].

A noteworthy artefact of our study was that a large proportion of the cohort (over 60\%) demonstrated or discovered enthusiasm for self-monitoring (i.e. tracking). In the course of the longitudinal tracking, 13 participants decided they would like to wear the wrist activity tracker continuously and not just for the durations of data collection. Nine participants either already possessed their own tracking device (such as a Fitbit ${ }^{4}$, Apple Watch ${ }^{5}$, Android Watch, or phone step counter app) or decided to purchase one, based on experiencing self-monitoring while volunteering for the project. This willingness to self-monitor using activity-tracking technologies indicates that participants find value and recognise benefits that digital health aids bring. This is another point that requires a great deal of cautious interpretation, as it is constrained by the self-selecting nature of our sample. According to the Technology Adoption Life Cycle model [19] our sample likely consists of a mixture of early adopters and innovators- i.e. people who have the propensity to engage more enthusiastically with technological innovation or even contribute to its development. We had no access to harder to reach groups, e.g. older adults who may live in rural locations, are not too active and may lead an altogether more isolated life. The TALC model proposes that this population may be the so-called laggards/phobics individuals reluctant to adopt technologies.

\footnotetext{
${ }^{4}$ https://www.fitbit.com/uk/home
}

\subsection{Strengths and limitations}

The present study had several strong points. Methodologically triangulated data was one of them. We used well-established, validated scales, which provided a standardised protocol for each time point. The use of diaries was another successful data collection technique, with which participants engaged well. It is important because it explores what people actually do to stay healthy rather than simply their stated preferences for activities which may vary from their actual behaviour [22]. Finally, activity tracking provided validation to participants' own perceptions on involvement in physical activity.

The present study has several methodological limitations. Longitudinal tracking requires a certain amount of time commitment from participants and attrition rates are typically a problem for studies like ours. While we recruited 35 participants initially, we were only able to test between 70 and $80 \%$ of them at any given time point. And while an attrition rate of around $20 \%$ is to be anticipated, for a small sample like ours this can be quite problematic, therefore caution is advised in the interpretation of the results. However, the key uncertainty with which we are left after the present study has to do with generalizing our findings beyond the cohort we accessed and to populations of lower socio-economic status and lower to no engagement with activities and technologies. Future research may also wish to explore combining activity tracking with means for self-management of age related illness and disability, as they are an almost inevitable part of normal ageing and impact daily functioning of older adults.

A further limitation of this research was the use of aggregate data to explore the relationships between the variables. This was to offer statistical significance to the relationships we were investigating. This provides the basis for understanding sources of wellbeing more generally, but in further work we want to explore individual variations in sources of wellbeing as we gather more data. This will further help us to identify ways of promoting accessible wellbeing.

\subsection{How quantified successful ageing was used as a design resource for accessible wellbeing recommendations}

There are many different ways of designing technology for wellbeing for older adults. One could conduct focus groups or workshops to ask older adults about the health issues that they want to track [18]. That could focus attention on areas of concern for older adults such as social interaction tracking, stress relief activities, or eating tracking. Another approach would be to administer questionnaires to find the topics older adults are interested in [11] - topics such as photos, recipes and bank statements. Still another approach would be to circumvent conscious input from the user and use ambient sensors to detect signals of wellbeing change [25]. Our approach is somewhat different. We suggest that older adults can be actively involved in the process of collecting data about their lives - something that they are often interested in - and that this data can be used as a design resource to understand what areas of their lives to target with

\footnotetext{
${ }^{5}$ https://www.apple.com/uk/watch/
} 
support and intervention. For example, in this study we saw the importance for wellbeing of meeting with people from interest groups (such as a reading group or acting group). By monitoring such activities and designing to encourage such activities, we deliberately target what we know is beneficial for wellbeing and accessible to a range of older adults.

Furthermore, the usefulness of longitudinal data for understanding wellbeing provides the basis for using that data in a personalized way in a recommender system. While we have considered aggregate data here, our system will offer personalized recommendations for users based on their own sources of wellbeing. While here we have pointed out sources of wellbeing in general, it may be that different users have different predictors of wellbeing and our system can monitor these predictors, identify their effects, and recommend accessible activities accordingly.

Thus our social network is designed to encourage social participation and our findings support the notion of the central role of social activities in ageing well. The hypothesised mechanisms of impact of the system suggest that invitations for social activities will result in increased physical activity and ultimately improvements in quality of life and wellbeing. The system could provide opportunities for self-monitoring and behavioural tracking, to reward and reinforce physical activity efforts. Behavioural patterns such as completing one's shopping on a Saturday, gardening on Sunday and exercise on week days should be recognized and embedded within the recommendations, in order for the system to work in synchrony with users' habits.

\subsection{Future work}

Having identified the behavioural principles that facilitate successful behaviour change (easy, attractive, social, and timely [27]), we have been able to focus on the kinds of activities that will be easy and attractive to users, will involve their social networks, and will be temporally suitable. In developing a recommender system around this, we propose several requirements:

1. The system should use both a content-based and collaborative filtering algorithm to recommend events to users consistent with previous research [15, 20, 37]. The content-based approach is suitable because often the events users identified as being engaged in are repeated events such as attending activity groups, classes or going to religious groups. The collaborative filtering approach is also suitable because it is through friendships that users are likely to find out about other interesting groups. This satisfies the requirement for an easy and attractive approach to behaviour change.

2. The recommender system should draw on social network data about users to understand when group members are planning meetings, and to facilitate organization of meetings between group members. This draws on the insight that meeting with other interest-group members has distinct benefits for wellbeing. We are already working on testing UI mockups for such a system.

3. The recommendations from the system must be timely. Not all events are suitable at all times. Using machine-learning, the system should understand when the user is most likely to leave home or attend events with friends. It should also distinguish between different times of the year (particularly relevant in countries where distinct seasons render some outdoor activities impossible) in order to provide these recommendations. For example, encouraging a walking group to meet for a walk on a wet winter day would not be appropriate, but recommending such an activity in the spring or summer could be timely and appropriate.

In identifying these requirements, we aim to make explicit the connections between this user research and the design of the system so that it is more effective in producing positive change in the lives of the users.

\subsection{Conclusions}

In this study we tracked the trajectory of a group of older adults for 18 months. Our results suggest that social and physical activity are key factors for successful ageing and are implicated in the prevention of age-related decline in functioning. In addition, our findings suggest that social and physical activity are closely linked and positively impacting one of these activities is likely to affect the other in the same way. In conclusion, these results provide insight into how to use data from quantified older adults as a design resource to understand what benefits them most in order to design a system that seeks to offer accessible recommendations to create maximal benefits.

\section{ACKNOWLEDGMENTS}

We thank all the volunteers who have contributed to this research. This project was funded by the European Union's Horizon 2020 research and innovation programme under grant agreement No 643644 (ACANTO: A CyberphysicAL social NeTwOrk using robot friends).

\section{REFERENCES}

[1] Almeida, O.P. and Almeida, S. a. 1999. Short versions of the Geriatric Depression Scale: A study of their validity for the diagnosis of a major depressive episode according to ICD-10 and DSM-IV. International Journal of Geriatric Psychiatry. 14, 10 (1999), 858-865. DOI:https://doi.org/10.1002/(SICI)1099-1166(199910)14:10<858::AIDGPS35>3.0.CO;2-8.

[2] Apter, M.J. 1984. Reversal theory and personality: A review. Journal of Research in Personality. 18, 3 (1984), 265-288. DOI:https://doi.org/10.1016/0092-6566(84)90013-8.

[3] Attick, J. et al. 2016. Wearable Technology and Older Adult Motivation : Urban versus Suburban Communities. (2016), 1-6.

[4] Baker, L.D. et al. 2010. Effects of Aerobic Exercise on Mild Cognitive Impairment. Archives of Neurology. 67, 1 (Jan. 2010), 71-79. DOI:https://doi.org/10.1001/archneurol.2009.307.

[5] Baumeister, R.F. and Leary, M. 1995. The need to belong: Desire for interpersonal attachments as a fundamential human motivation. Psychology and Aging. 117, 3 (1995), 497-529.

[6] Beauchet, O. et al. 2014. Quantified self and comprehensive geriatric assessment: Older adults are able to evaluate their own health and functional status. PLoS ONE. 9, 6 (2014), 1-5. DOI:https://doi.org/10.1371/journal.pone.0100636.

[7] Bennett, G.G. et al. 2011. The Association Between Social Factors and Physical Activity Among Low-Income Adults Living in Public Housing. American Journal of Public Health. 101, 11 (2011), 2102-2110. DOI:https://doi.org/10.2105/ajph.2010.196030.

[8] Bruine de Bruin, W. et al. 2016. HHS Public Access. 31, 3 (2016), 1-21. DOI:https://doi.org/10.1021/acschemneuro.5b00094.Serotonin.

[9] Burke, R. 2007. Hybrid Web Recommender Systems. The Adaptive Web. Springer Berlin Heidelberg. 377-408. 
[10] Cacioppo, J.T. and Cacioppo, S. 2014. Social Relationships and Health: The Toxic Effects of Perceived Social Isolation. Soc Personal Psychol $\begin{array}{llll}\text { Compass. } & 8, & 2 & \text { (2014), }\end{array}$ DOI:https://doi.org/10.1111/spc3.12087.Social.

[11] Caprani, N. et al. 2010. I like to log: A Questionnaire Study towards Accessible Lifelogging for Older Users. Proceedings of the 12th international ACM SIGACCESS conference on Computers and accessibility - ASSETS '10 (New York, New York, USA, 2010), 263.

[12] Carstensen, L.L. et al. 2011. Emotional experience improves with age: Evidence based on over 10 years of experince sampling. Psychology of Aging. 26, 1 (2011), 21-33. DOI:https://doi.org/10.1037/a0021285.Emotional.

[13] Carstensen, L.L. et al. 2000. Emotional experience in everyday life across the adult life span. Journal of Personality and Social Psychology. 79, 4 (2000), 644-655. DOI:https://doi.org/10.1037/0022-3514.79.4.644.

[14] Charles, S.T. et al. 2001. Age-related differences and change in positive and negative affect over 23 years. Journal of Personality and Socia Psychology. 80, 1 (2001), 136-151. DOI:https://doi.org/10.1037//00223514.80.1.136.

[15] Cornelis, C. et al. 2005. A Fuzzy Relational Approach to Even Recommendation. Indian International Conference on Artificial Intelligence. October (2005), 2231-2242.

[16] Cornwell, B. and Laumann, E.O. 2015. The health benefits of network growth: New evidence from a national survey of older adults. Socia Science and Medicine. 125, (2015), 94-106. DOI:https://doi.org/10.1016/j.socscimed.2013.09.011.

[17] Dall, P.M. et al. 2017. The influence of dog ownership on objective measures of free-living physical activity and sedentary behaviour in community-dwelling older adults: A longitudinal case-controlled study. BMC Public Health. 17, 1 (2017), 1-9. DOI:https://doi.org/10.1186/s12889-017-4422-5.

[18] Davidson, J.L. and Jensen, C. 2013. What health topics older adults want to track. Proceedings of the 15th International ACM SIGACCESS Conference on Computers and Accessibility - ASSETS '13 (New York, New York, USA, 2013), 1-8.

[19] Diederen, P. et al. 2003. Innovation Adoption in Agriculture : Innovators Early Adopters and Laggards. Cahiers d'économie et sociologie rurales. 67 (2003), 30-50.

[20] Dooms, S. et al. 2011. A user-centric evaluation of recommender algorithms for an event recommendation system. CEUR Workshop Proceedings. 811, (2011), 67-73. DOI:https://doi.org/10.1063/1.3362135.

[21] Engel, G. 1980. The clinical application of the biopsychosocial model. American Journal of Psychiatry. 137, 5 (May 1980), 535-544. DOI:https://doi.org/10.1176/ajp.137.5.535.

[22] Eyal, N. 2014. Hooked: How to build habit-forming products. Penguin.

[23] Fisken, A. et al. 2015. Perceived benefits, motives, and barriers to aquabased exercise among older adults with and without osteoarthritis. Journa of Applied Gerontology. 34, 3 (2015), 377-396. DOI:https://doi.org/10.1177/0733464812463431.

[24] Fox, S. and Duggan, M. 2013. Health online 2013. Health. (2013), 1-55. DOI:https://doi.org/2013/01/15/health-online-2013/.

[25] Gil, N.M. et al. 2007. Data visualisation and data mining technology for supporting care for older people. Proceedings of the 9th international ACM SIGACCESS conference on Computers and accessibility - Assets '07 (New York, New York, USA, 2007), 139.

[26] Grieve, F.G. and Vander Weg, M.W. 2003. Desire to eat high- and low-fat foods following a low-fat dietary intervention. Journal of nutrition education and behavior. 35, 2 (2003), 98-102.

[27] Halpern, D. 2016. Inside the nudge unit: How small changes can make a big difference. Random House.

[28] Haslam, C. et al. 2010. The social treatment: The benefits of group interventions in residential care setting. Psychology and Aging. 25, 1 (2010), 157-167. DOI:https://doi.org/10.1037/a0018256.

[29] Hawkley, L.C. and Cacioppo, J.T. 2010. Loneliness Matters: A Theoretical and Empirical Review of Consequences and Mechanisms. Annals of Behavioral Medicine. 40, 2 (Oct. 2010), 218-227. DOI:https://doi.org/10.1007/s12160-010-9210-8.

[30] Hawley, G. et al. 2008. Sustainability of health and lifestyle improvements following a non-dieting randomised trial in overweight women. Preventive $\begin{array}{llll}\text { medicine. } & 47, & 6 & \text { (2008), }\end{array}$ DOI:https://doi.org/http://dx.doi.org/10.1016/j.ypmed.2008.08.008.

[31] Herdman, M. et al. 2011. Development and preliminary testing of the new five-level version of EQ-5D (EQ-5D-5L). Quality of Life Research. 20, 10 (2011), 1727-1736. DOI:https://doi.org/10.1007/s11136-011-9903-x.

[32] International Wellbeing Group 2013. Personal Wellbeing Index: 5th Edition. Australian Centre on Quality of Life, Deakin University.

[33] Jetten, J. et al. 2009. The Social Cure. Scientific American Mind. 20, 5 (2009), 26-33. DOI:https://doi.org/10.1038/scientificamericanmind0909-
26.

[34] Kempen, G.I.J.M. et al. 2008. The Short FES-I: A shortened version of the falls efficacy scale-international to assess fear of falling. Age and Ageing. 37, 1 (2008), 45-50. DOI:https://doi.org/10.1093/ageing/afm157.

[35] Kerr, J. et al. 2012. The relationship between outdoor activity and health in older adults using GPS. International Journal of Environmental Research and Public Health. 9, 12 (2012), 4615-4625. DOI:https://doi.org/10.3390/ijerph9124615.

[36] Kessler, E.M. and Staudinger, U.M. 2009. Affective Experience in Adulthood and Old Age: The Role of Affective Arousal and Perceived Affect Regulation. Psychology and Aging. 24, 2 (2009), 349-362. DOI:https://doi.org/10.1037/a0015352.

[37] Khrouf, H. and Troncy, R. 2013. Hybrid event recommendation using linked data and user diversity. Proceedings of the 7th ACM conference on Recommender systems - RecSys '13. (2013), 185-192. DOI:https://doi.org/10.1145/2507157.2507171.

[38] Lehman, B.J. et al. 2017. Rethinking the biopsychosocial model of health: Understanding health as a dynamic system. Social and Personality Psychology Compass. 11, 8 (Aug. 2017), e12328. DOI:https://doi.org/10.1111/spc3.12328.

[39] Liu, X. et al. 2012. Event-based social networks. Proceedings of the 18th ACM SIGKDD international conference on Knowledge discovery and data mining - KDD '12 (New York, New York, USA, Dec. 2012), 1032.

[40] McAuley, E. et al. 2000. Social Relations, Physical Activity, and WellBeing in Older Adults. Preventive Medicine. 31, 5 (2000), 608-617. DOI:https://doi.org/10.1006/pmed.2000.0740.

[41] McConnell, A.R et al. 2011. Friends with benefits: On the positive consequences of pet ownership. Journal of Personality and Social $\begin{array}{lllll}\text { Psychology. } & 101, & 6 & \text { (2011), } & 1239-1252 .\end{array}$ DOI:https://doi.org/10.1037/a0024506.

[42] McMurdo, M.E.T. et al. 2012. Social, Environmental and Psychologica Factors Associated with Objective Physical Activity Levels in the Over 65s. PLoS ONE. 7, 2 (Feb. 2012), e31878. DOI:https://doi.org/10.1371/journal.pone.0031878.

[43] Mcnicholas, J. et al. 2005. Evidence and issues. BMJ: British Medical Journal. 331, 7527 (2005), 1252-1254.

[44] Morrison, S. et al. 2016. Walking-Induced Fatigue Leads to Increased Falls Risk in Older Adults. Journal of the American Medical Directors $\begin{array}{lllll}\text { Association. } & 17, & 5 & \text { (2016), } & 402-409\end{array}$ DOI:https://doi.org/10.1016/j.jamda.2015.12.013.

[45] Paul, S.S. et al. 2015. Validity of the Fitbit activity tracker for measuring steps in community-dwelling older adults. BMJ Open Sport \& Exercise Medicine. 1, 1 (2015), e000013. DOI:https://doi.org/10.1136/bmjsem2015-000013.

[46] Peel, C. et al. 2005. Assessing mobility in older adults: the UAB Study of Aging Life-Space Assessment. Physical therapy. 85, 10 (2005), 1008-119.

[47] Rantakokko, M. et al. 2009. Fear of moving outdoors and development of outdoor walking difficulty in older people: Clinical Investigations. Journal of the American Geriatrics Society. 57, 4 (2009), 634-640. DOI:https://doi.org/10.1111/j.1532-5415.2009.02180.x.

[48] Rejeski, W.J. and Mihalko, S.L. 2017. Physical Activity and Quality of Life in Older Adults. 56, Ii (2017), 23-35. DOI:https://doi.org/10.1001/jama.1997.03540250072035.

[49] Rivas, H. and Wac, K. 2017. Digital Health.

[50] Smith, B.W. et al. 2008. The brief resilience scale: assessing the ability to bounce back. International journal of behavioral medicine. 15, 3 (2008), 194-200. DOI:https://doi.org/10.1080/10705500802222972.

[51] Strawbridge, W.J. et al. 2002. Physical activity reduces the risk of subsequent depression for older adults. American Journal of Epidemiology. 156, 4 (2002), 328-334. DOI:https://doi.org/10.1093/aje/kwf047.

[52] Studenski, S. et al. 2014. Gait Speed and Survival in Older Adults. 305, 1 (2014).

[53] Swan, M. 2012. Health 2050: The realization of personalized medicine through crowdsourcing, the quantified self, and the participatory biocitizen. Journal of Personalized Medicine. 2, 3 (2012), 93-118. DOI:https://doi.org/10.3390/jpm2030093.

[54] Tudor-locke, C. et al. 2011. How Many Steps / day are Enough ? For Adults. International Journal of Behavioral Nutrition and Physical Activity 2011. (2011), 1-17. DOI:https://doi.org/10.1186/1479-5868-8-79.

[55] Watson, D. et al. 1988. Development and validation of brief measures of positive and negative affect: The PANAS scales. Journal of Personality and Social Psychology. 54, 6 (1988), 1063-1070. DOI:https://doi.org/10.1037/0022-3514.54.6.1063.

[56] Yesavage, J. et al. 1983. Development and validation of a geriatric depression screening scale: a preliminary report. Journal of psychiatric research. 17, 1 (1983), 37-49. DOI:https://doi.org/10.1016/00223956(82)90033-4. 